\title{
Prospecção Tecnológica sobre a Utilização do Stryphnodendron Adstringens (Mart.) Coville (Barbatimão)
}

\author{
Technological Prospection on the Use of Stryphnodendron Adstringens \\ (Mart.) Coville (Barbatimão)
}

\author{
Cristiane Araujo Nascimento ${ }^{1}$ \\ Tatiane Luciano Ballian ${ }^{1}$ \\ Denise Macêdo Silva ${ }^{1}$ \\ Renan Macêdo Silva ${ }^{1}$ \\ Karol Fireman Farias ${ }^{1}$ \\ Guilherme Benjamin Brandão Pitta ${ }^{1}$ \\ ${ }^{1}$ Universidade Federal de Alagoas, Maceió, AL, Brasil
}

\begin{abstract}
Resumo
O Stryphnodendron adstringens (MART.) Coville é uma planta medicinal, popularmente conhecida como barbatimão $e$ amplamente valorizada por suas propriedades medicinais devido à alta quantidade de taninos, assim como, de outros constituintes químicos. O presente trabalho teve por objetivo realizar uma prospecção tecnológica sobre a utilização do Stryphnodendron adstringens (MART.) Coville (barbatimão). As buscas de patentes foram realizadas nos bancos de dados do Instituto Nacional de Propriedade Industrial (INPI), European Patent Office (EPO), United States Patent and Tradmark Office (USPTO) e World Intelectual Property Organization (WIPO). Foram analisadas 18 patentes, das quais $88,8 \%$ eram pertencentes à seção A Classificação Internacional de Patentes (IPC), referente às necessidades humanas. Os anos de 2003, 2006 e 2010 apresentaram o maior número de registros. O Brasil revelou-se como o maior detentor de depósitos de patentes com $94,45 \%$ dos documentos. A partir do mapeamento tecnológico realizado neste estudo, foi possível observar que a presente espécie se apresenta como um favorável seguimento de mercado, principalmente, do ponto de vista farmacológico.
\end{abstract}

Palavras-chave: Stryphnodendron adstringens (MART.) Coville. Barbatimão. Prospecção tecnológica.

\begin{abstract}
Stryphnodendron adstringens (MART.) Coville is a medicinal plant, popularly known as barbatimão and widely valued for its medicinal properties due to the high amount of tannins as well as other chemical constituents. The present work aimed to carry out a technological prospection on the use of Stryphnodendron adstringens (MART.) Coville (barbatimão). The patent searches were carried out in the INPI, EPO, USPTO and WIPO databases. A total of 18 patents were analyzed, of which $88.8 \%$ belonged to Section A (IPC), referring to human needs. The years 2003 , 2006 and 2010 presented the highest number of registrations. Brazil presented itself as the largest holder of patent deposits with $94.45 \%$ of the documents. From the technological mapping carried out in this study, it was possible to observe that the present species presents itself as a favorable market follow-up, mainly from the pharmacological point of view.
\end{abstract}

Keywords: Stryphnodendron adstringens (MART.) Coville. Barbatimão. Technological prospecting.

Área tecnológica: Inovação e desenvolvimento. Tecnologia. Inovação em saúde. 


\section{Introdução}

O Stryphnodendron adstringens (MART.) Coville é uma planta medicinal, popularmente conhecida como barbatimão, barba-de-timão, chorãozinho-roxo, casca da virgindade ou casca-da-mocidade, típica do cerrado e distribuída na maioria do território brasileiro (BORGES FILHO; FELFILI, 2003; TAKARNIA; DOBEREINER; PEIXOTO, 2000; LORENZI; MATOS, 2008). Sua alta concentração de taninos e de outros componentes químicos, como, alcaloides, inibidores de proteases (como a tripsina), flavonoides e esteroides são responsáveis por promover a essa espécie propriedades únicas para aplicação na área medicinal (MACEDO et al., 2007).

Considerando-se que os taninos são o principal componente do barbatimão, cuja concentração pode variar de 20 a $50 \%$, cujo tipo mais presente é os taninos condensados, que contêm unidades de prodelfinidina, podendo ser galocatequina e/ou epigalocatequina (LOPES et al., 2005; LIMA et al., 2010; LOPES et al., 2009). Sua habilidade para formar complexos insolúveis em água com proteínas proporciona atividades biológicas como efeito adstringente, cicatrizante, antifúngico, anti-inflamatório, antimicótico e antisséptico, tornando-se assim, uma substância extremamente atrativa para a indústria farmacêutica e o uso popular (SANTOS et al., 2002; MELLO; PETEREIT; NAHRSTEDT, 1996).

Na prática de saúde, o barbatimão tem algumas de suas ações comprovadas por meio do uso popular, como a melhora de corrimentos vaginais, hemorragias e infecções uterinas, feridas cutâneas, inflamações de garganta, entre outros (SANTOS; SEBASTIANI, 2011; BRANDÃO et al., 2009). Nessa perspectiva, pesquisas vêm sendo desenvolvidas com abordagem clínica, química e biofarmacêutica, a fim de comprovar sua ação farmacológica por meio de evidências científicas, além de avaliar efeitos como a toxicidade (FERREIRA; SILVA, 2013). Dessa forma, o barbatimão apresenta-se como uma planta com propriedades de caráter inovador, com alto valor comercial e medicinal, além de apresentar um grande número de aplicações (BORGES FILHO; FELFILI, 2003).

Nesse contexto, o presente trabalho teve por objetivo realizar uma prospecção tecnológica sobre a utilização do Stryphnodendron adstringens. (MART.) Coville (barbatimão) por meio da análise sistemática de patentes e elaboração de um panorama geral no mundo sobre a temática.

\section{Metodologia}

Esta prospecção foi realizada com base nos pedidos de patentes depositados nos bancos de dados do Instituto Nacional de Propriedade Industrial (INPI), European Patent Office (EPO), United States Patent and Tradmark Office (USPTO) e World Intelectual Property Organization (WIPO) (Figura 1). A pesquisa processou-se mediante o levantamento e análise de documentos de patentes, com abordagem quantitativa e exploratória. Foram utilizados os termos de busca: Stryphnodendron Adstringens, Stryphnodendron Adstringens (MART.) Coville e barbatimão, considerando todos os documentos que apresentaram esses termos no título e resumo. As variáveis foram escolhidas de acordo com a disponibilidade dos dados, das quais foram analisadas: Classificação Internacional de Patentes (IPC), país, ano de publicação, inventores, depositantes e procurador. 
Os dados foram tabulados e organizados nos softwares Microsoft Excel 2010 e Microsoft Word 2010. Para a construção de gráficos e figuras empregou-se o CmapTools version 5.00 e Microsoft Excel 2010.

Figura 1 - Metodologia utilizada para pesquisa e seleção de patentes em base de dados

\section{Estratégia de busca}

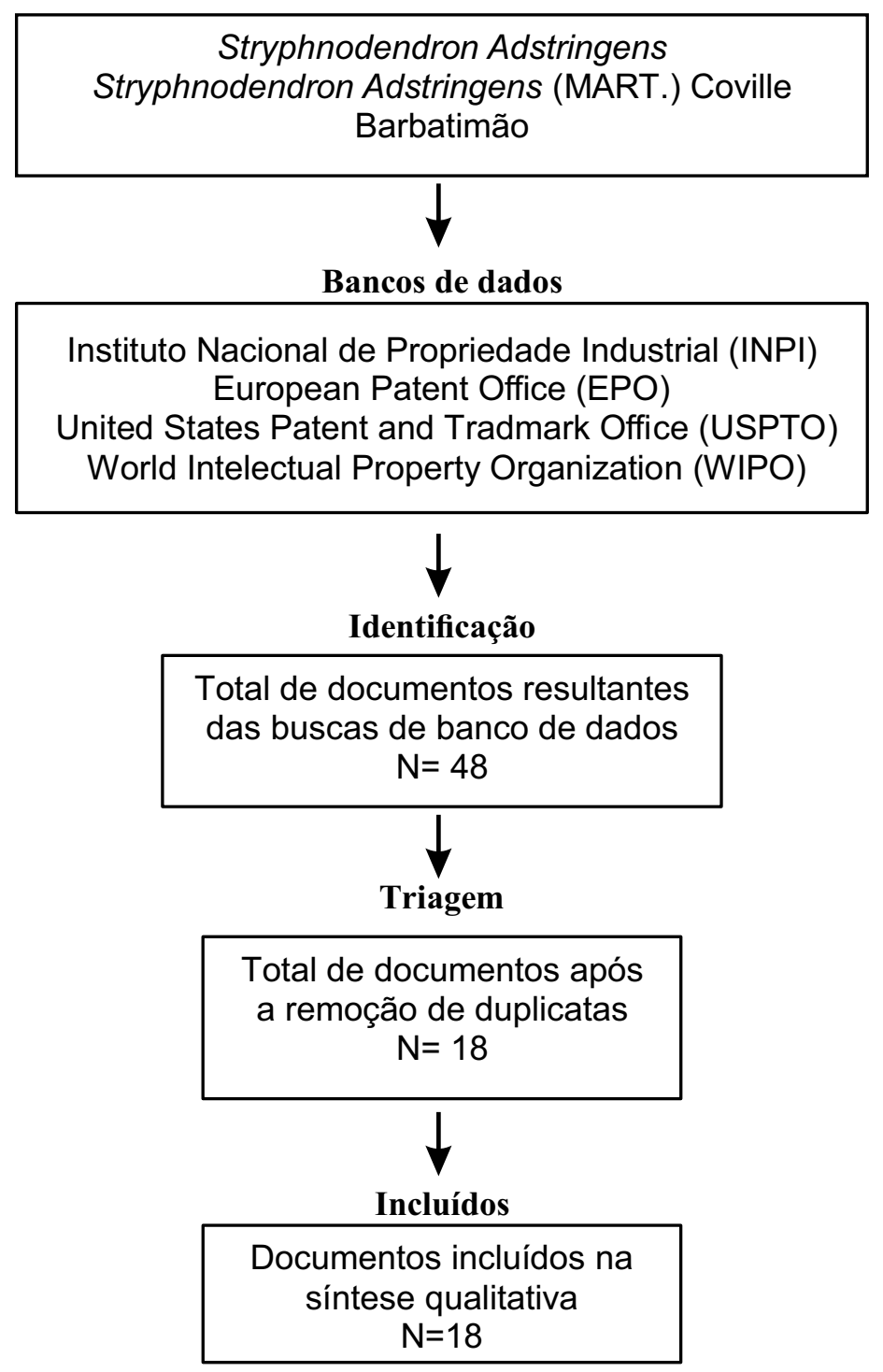

Fonte: Elaborada pelos autores deste artigo (2018)

\section{Resultados e Discussão}

Foram encontradas 48 patentes referentes ao uso do Stryphnodendron adstringens (MART.) Coville, dessas, 29 foram removidas por tratar-se de duplicatas, restando 18 documentos para análise detalhada. Os resultados do número de patentes encontrado nos bancos de dados consultados estão apresentados na Tabela 1. Destacando-se o banco de dados do INPI e WIPO com 25 e 22 resultados, respectivamente. O número semelhante de registros em ambas as bases, demonstra a preocupação dos autores em fazer o depósito em mais de uma base, a fim de melhor proteger sua invenção. 
Tabela 1 - Número de patentes depositadas nas bases de dados INPI, WIPO, EPO e USPTO de acordo com os termos de busca

\begin{tabular}{|c|c|c|c|c|}
\hline \multirow{2}{*}{ Termo DE BusCa } & \multicolumn{4}{|c|}{ Base de Dados } \\
\hline & INPI & WIPO & EPO & USPTO \\
\hline Stryphnodendron Adstringens & 09 & 08 & 0 & 01 \\
\hline $\begin{array}{c}\text { Stryphnodendron Adstringens. } \\
\text { (MART.) Coville }\end{array}$ & 01 & 02 & 0 & 0 \\
\hline Barbatimão & 15 & 12 & 0 & 0 \\
\hline
\end{tabular}

Fonte: Elaborada pelos autores deste artigo (2018)

No presente estudo a IPC teve maior concentração de número de depósitos na seção A (Necessidades humanas) com 16 patentes, seguido da subseção A61 (Ciência médica ou veterinária; higiene) com 10 resultados. O código A61K referente a Preparações para finalidades médicas, odontológicas ou higiênicas esteve presente em sete das patentes identificadas. Os códigos completos mais frequentes foram A61K 36/48 $(n=5)$ relacionadas a Preparações medicinais contendo materiais de constituição indeterminadas derivados de algas, líquens, fungos ou plantas, ou derivados do Fabaceae ou Leguminosae (família da ervilha e do legume); Caesalpiniaceae; Mimosaceae; Papilionaceae, e A61K 8/97 (n=2) referente a Cosméticos ou preparações similares para higiene pessoal derivados de algas, líquens, fungos ou plantas. Em relação à seção C (Química; Metalurgia) foram encontradas duas patentes pertencentes as subseções C11D 9/00 relacionada a composições de detergentes baseados essencialmente em saponáceos e C09J 199/00 referente a adesivos à base de compostos macromoleculares naturais ou de seus derivados (Figura 2).

Figura 2 - Número de patentes depositadas nas bases de dados INPI, WIPO, EPO e USPTO de acordo com os termos de busca

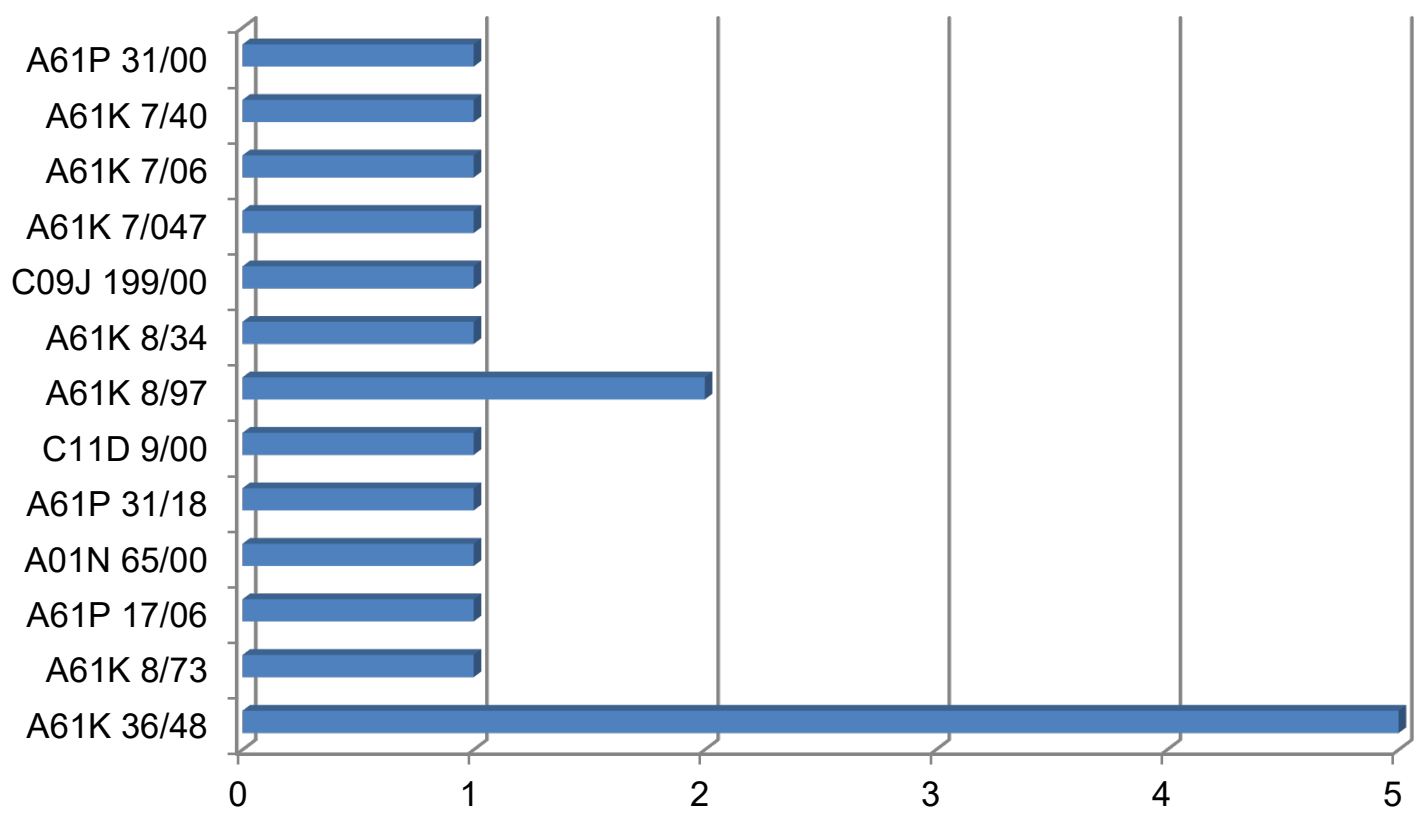

Fonte: Elaborada pelos autores deste artigo (2018) 
Ao analisar a evolução anual dos depósitos de patentes, constatou-se que o primeiro registro de depósito de patente referente ao uso da espécie Stryphnodendron adstringens (MART.) Coville ocorreu em 1997, no estado brasileiro de São Paulo. A patente tratava de elementos terapêuticos e processo para tratamento de psoríase e suas lesões. O maior número de depósitos ocorreu nos anos de 2003, 2006 e 2010, com três registros em cada ano. O último depósito de patente registrado ocorreu em 2016 (Figura 3).

Figura 3 - Distribuição das patentes de acordo com o ano de depósito

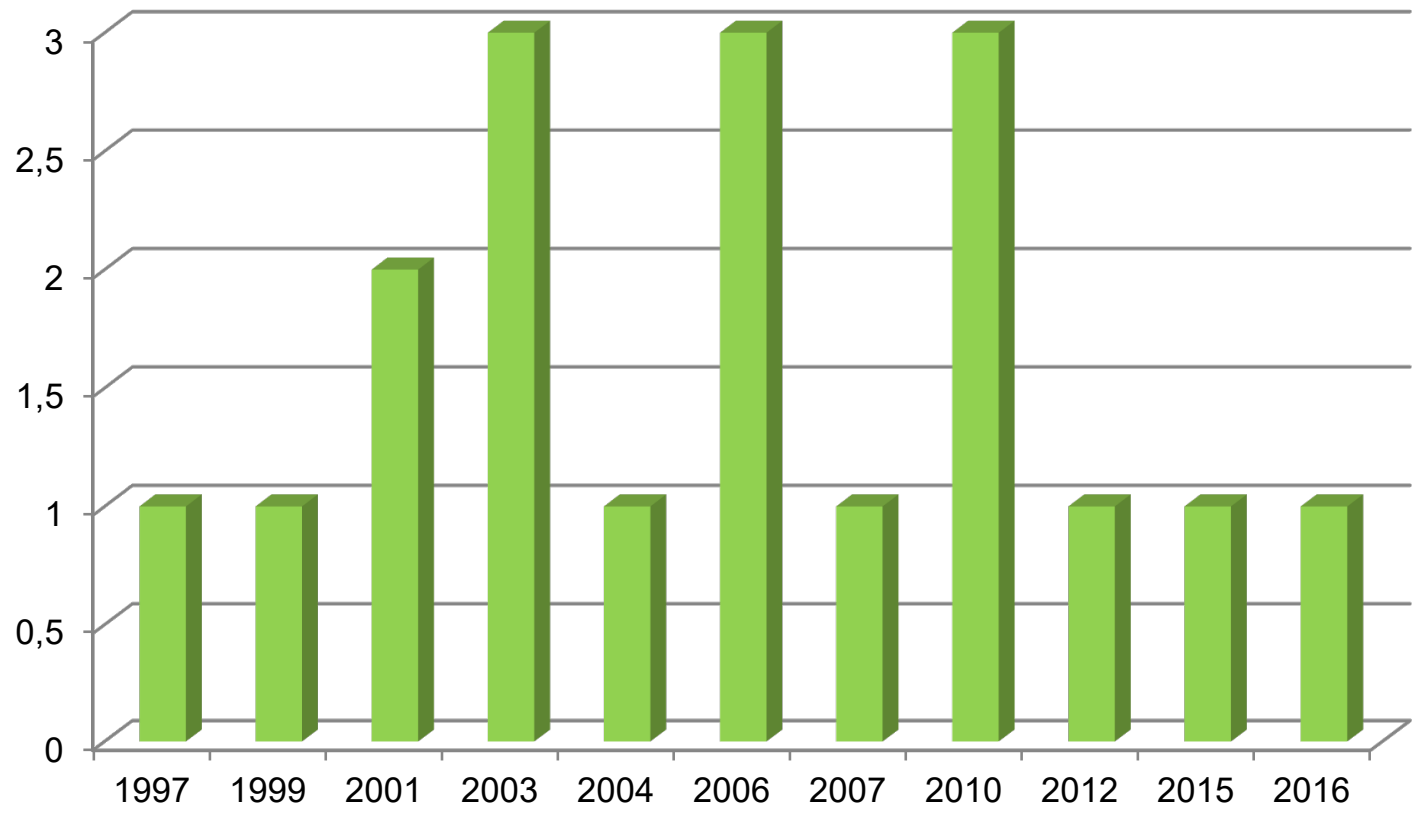

Fonte: Elaborada pelos autores deste artigo (2018)

Ao observar os resultados de acordo com o país de depósito das patentes, constatou-se que o Brasil é detentor de $94,45 \%(n=17)$ dos documentos de patentes e o Japão é responsável por $5,55 \%(n=1)$ dos depósitos (Figura 3). Dessa forma, pode-se afirmar o grande interesse em pesquisa e produção de produtos, principalmente, farmacêuticos à base da espécie Stryphnodendron adstringens (MART.) Coville (barbatimão) no Brasil, comparado a outros países.

Tais resultados estão de acordo com a literatura, onde é evidenciada a grande diversidade de espécies vegetais com propriedades medicinais e a busca por novas alternativas de tratamento através dessas plantas no Brasil (SILVA; MARINI; MELO, 2015). O fortalecimento do uso de plantas medicinais no Brasil se deu, principalmente, por meio da criação da Política Nacional de Práticas Integrativas e Complementares (PNPIC) e da Política Nacional de Plantas Medicinais e Fitoterápicos, ambas criadas no ano de 2006. Em 2009, foi publicada a Relação Nacional de Plantas Medicinais de Interesse ao SUS (RENISUS), onde estão descritas diferentes plantas medicinais, inclusive o barbatimão, tal fato fortaleceu ainda mais o uso dessa planta (FIGUEREDO; GURGEL; GURGEL JUNIOR, 2014). 
Figura 4 - Número de depósitos de patentes de acordo com o país

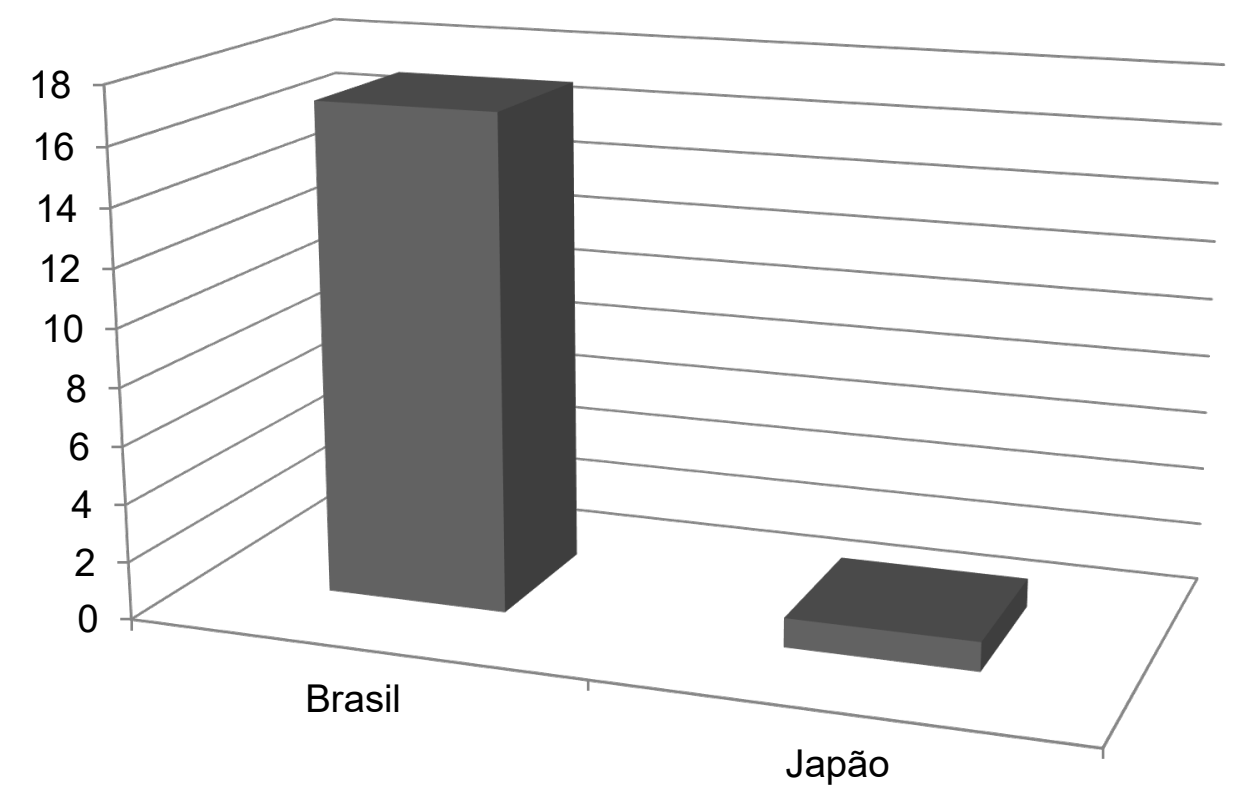

Fonte: Elaborada pelos autores deste artigo (2018)

Em relação ao número de depósitos de patentes realizado por cada inventor, todos obtiveram a mesma quantidade de documentos depositados. O maior número de inventores de patentes foram pessoas físicas $(n=17)$, seguido de apenas uma instituição de ensino superior $(n=1)$ (Figura 4). Porém, ao analisar a frequência em que estes inventores apareceram em cada banco de dados com a mesma invenção, destacaram-se os inventores Manoel Álvaro de Freitas Lins Neto e colaboradores, cuja patente de invenção intitulada "Composição Farmacêutica para Tratamento de Infecções HPV utilizando Extratos de Barbatimão" foi a mais frequente durante as buscas, aparecendo 12 vezes.

A patente aborda o uso do barbatimão na forma de pomada no tratamento de infecções causadas pelo Papiloma Vírus Humano (HPV). Em 2010, foi depositada no INPI, e então, no ano de 2013 foi concedida pelo instituto americano The United States Patent and Trademark Office (US 9.023.405), cujo depósito internacional foi realizado via PCT. A pomada ainda não pode ser comercializada devido à necessidade de estudos clínicos e aprovação pela Agência Nacional de Vigilância Sanitária (ANVISA). Em contrapartida, a pomada Fitoscar, pertencente à empresa Apsen Farmacêutica S/A (CNPJ 62.462.015/000129) foi registrada na ANVISA e é indicada como agente cicatrizante em diferentes tipos de lesões. 
Figura 5 - Número de depósitos de patentes de acordo com o tipo de inventor

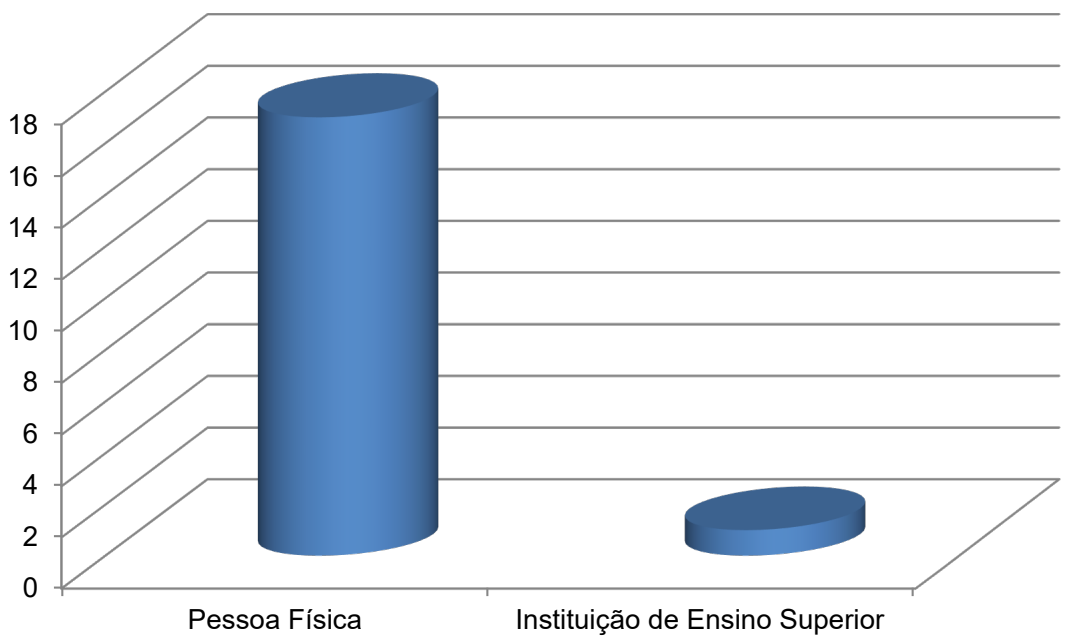

Fonte: Elaborada pelos autores deste artigo (2018)

Os depósitos de patentes foram realizados em maioria pelos próprios inventores, exceto em um dos depósitos, o qual foi realizado por uma Instituição de Ensino Superior (IES). Durante o levantamento dos dados, $55,5 \%(\mathrm{n}=10)$ dos depositantes vincularam procuradores as suas invenções, estando em igual quantidade procuradores na qualidade de empresas de marcas e patentes $(n=5)$ e pessoas físicas $(n=5)$. A empresa "Difusão Marcas e Patentes Ltda" consta como procuradora de duas patentes, os demais aparecem como procuradores de uma única patente (Figura 6).

A vinculação de procuradores vem se tornando frequente, especialmente, em casos cujo inventor possuí mais de um pedido de patente. Assim, o procurador responsabilizasse por prazos e trâmites legais de cada depósito realizado, podendo ser um procurador na forma de pessoa física, geralmente, um advogado, ou procurador na forma de pessoa jurídica que são empresas especializadas nesse segmento. Alguns inventores não fazem uso desse serviço, a fim de baratear o processo de registro de marcas e patentes (BOCCHINO et al., 2010).

Figura 6 - Distribuição dos procuradores das patentes

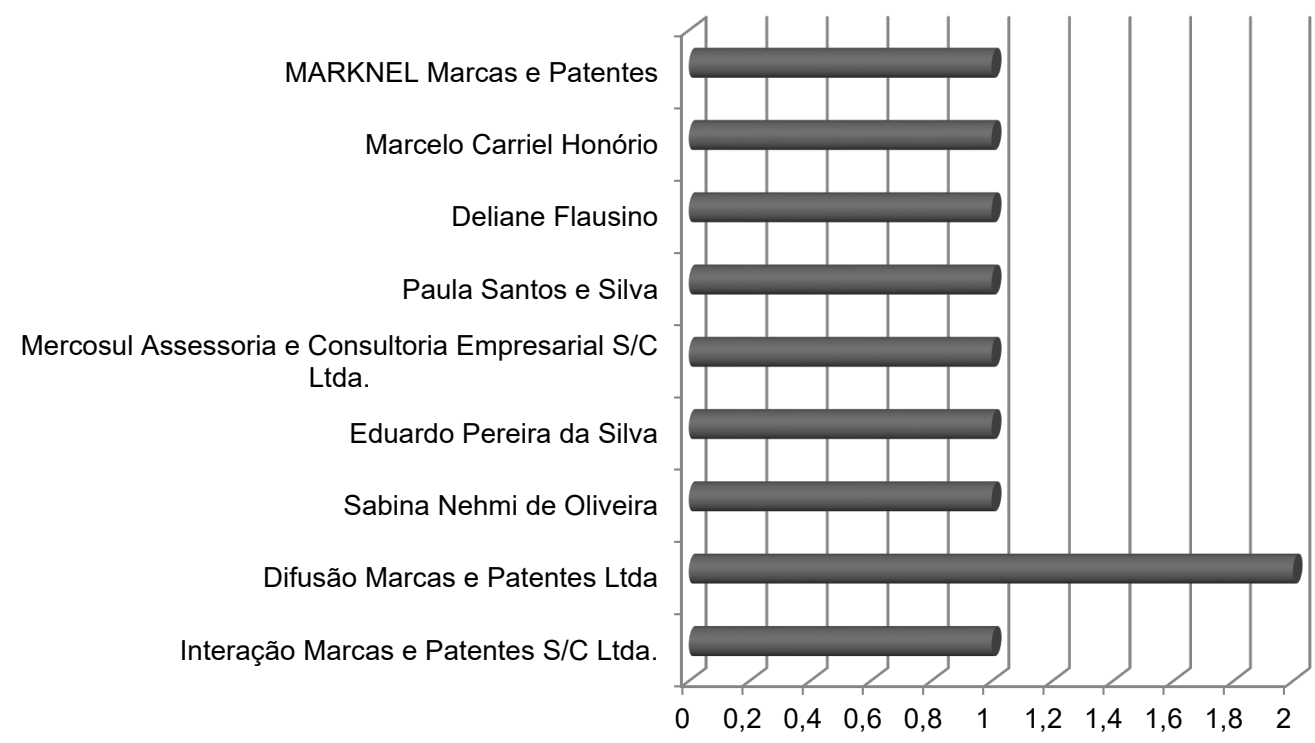

Fonte: Elaborada pelos autores deste artigo (2018) 


\section{Considerações Finais}

O mapeamento tecnológico realizado neste estudo evidenciou que a espécie Stryphnodendron adstringens (MART.) Coville (barbatimão) apresenta-se como um favorável seguimento de mercado, principalmente, do ponto de vista farmacológico. O Brasil vem se destacando no desenvolvimento de patentes sobre o barbatimão, configurando-se como o país com o maior número de depósitos, demonstrando assim, interesse na proteção de novos produtos desenvolvidos a base dessa planta medicinal, especialmente, por parte dos inventores e depositantes na forma de pessoa física, que representaram a maioria em ambos os casos.

A análise de perspectivas tecnológicas sobre o Stryphnodendron adstringens (MART.) Coville é fundamental para auxiliar na tomada de decisão e fortalecimento da competitividade de mercado. Salientando-se a importância do uso sustentável dessa planta medicinal, visto que, o barbatimão pode ser uma espécie ameaçada de extinção. Portanto, o barbatimão apresenta grande potencial de contribuição para o desenvolvimento da indústria nacional, desde que a biodiversidade seja preservada.

\section{Agradecimentos}

A Fundação de Amparo à Pesquisa do Estado de Alagoas (FAPEAL), à Coordenação de Aperfeiçoamento de Pessoal de Nível Superior (CAPES) e ao Programa de Pós-Graduação em Biotecnologia da Rede Nordeste de Biotecnologia (PPGB/RENORBIO) o apoio durante o desenvolvimento deste estudo.

\section{Referências}

BOCCHINO, L. O. et al. Propriedade Intelectual conceitos e procedimentos. Brasília, DF: Advocacia-Geral da União, 2010. 316 p.

BORGES FILHO, H. C.; FELFILI, J. M. Avaliação dos níveis de extrativismo da casca de barbatimão [Stryphnodendron adstringens (Mart.) Coville] no Distrito Federal, Brasil. Revista Árvore, Viçosa, MG, v. 27, n. 5, p. 735-745, 2003.

BRANDÃO, M. G. L. et al. Traditional uses of American plant species from the 1st edition of Brazilian Official Pharmacopoeia. Revista Brasileira de Farmacognosia, Curitiba, v. 19, n. 2, p. 478-487, 2009.

ESPACENET. Acesso gratuito a mais de $\mathbf{1 0 0}$ milhões de documentos de patentes. European Patent Office. [Base de dados - Internet]. 2018. Disponível em: <https://worldwide.espacenet. $\mathrm{com} />$. Acesso em: 13 maio 2018.

FERREIRA, E. C.; SILVA, J. L. L. As propriedades medicinais e bioquímicas da planta Stryphnodendron Adstringens barbatimão. Biológicas \& Saúde, [S.1.], v. 3, n. 11, dez. 2013.

FIGUEREDO, C. A.; GURGEL, I. G. D.; GURGEL JUNIOR, G. D. A Política Nacional de Plantas Medicinais e Fitoterápicos: construção, perspectivas e desafios. Physis, Rio de Janeiro, v. 24, n. 2, p. 381-400, 2014. 
INSTITUTO NACIONAL DA PROPRIEDADE INDUSTRIAL (INPI). [Base de dados - Internet]. 2018. Disponível em: < https://gru.inpi.gov.br/pePI/jsp/patentes/PatenteSearchAvancado.jsp>. Acesso em: 14 maio 2018.

LIMA, C. R. O. et al. Caracterização dos metabólitos secundários do barbatimão. In: SILVA, L. et al. Manual do Barbatimão. Goiânia: Kelps, 2010. cap.7, p.61-68. Manual do Barbatimão. Goiânia: Kelps, 2010. Cap.7, p.61-68.

LOPES, G. C. et al. Determinação quantitativa de taninos em três espécies de Stryphnodendron por cromatografia líquida de alta eficiência. Brazilian Journal of Pharmaceutical Sciences, São Paulo, v. 45, n. 1, p. 135-143, mar. 2009.

LOPES, G. C. et al. Influence of extracts of Stryphnodendron polyphyllum Mart. And Stryphnodendron obovatum Benth on the cicatrisation of cutaneous wounds in rats. Journal Ethnophamacol. [S.1.], v. 99, p. 265-272, 2005.

LORENZI, H.; MATOS, F. J. A. Plantas Medicinais no Brasil - Nativas e Exóticas. São Paulo: Instituto Plantarum, 2008.

MACEDO, F. M. et al. Triagem Fitoquímica do Barbatimão [Stryphnodendron adstringens (Mart) Coville]. Revista Brasileira de Biociências, Porto Alegre, v. 5, supl. 2, p. 1166-1168, jul. 2007.

MELLO, J. C. P.; PETEREIT, F; NAHRSTEDT, A. Prorobinetinidins from Stryphnodendron adstringens. Phytochemistry, Oxford, v. 42, n. 3, p. 857-862, jun. 1996.

SANTOS, S. C. et al. Tannin composition of barbatimão species. Fitoterapia, Amsterdan, v. 73, p. 292-299, 2002.

SANTOS, P. V.; SEBASTIANI, R. Plantas medicinais utilizadas por uma comunidade universitária no Município de São Paulo. Revista do Instituto de Ciências da Saúde, São Paulo, v. 29, n. 1, p. 11-15, 2011.

SILVA, M. D. P.; MARINI, F. S.; MELO, R. S. Levantamento de plantas medicinais cultivadas no município de Solânea, agreste paraibano: reconhecimento e valorização do saber tradicional.

Revista Brasileira de Plantas Medicinais, Campinas, v. 17, n. 4, supl. II, p. 881-890, 2015.

TAKARNIA, C. H.; DOBEREINER, J.; PEIXOTO, P. V. Plantas Tóxicas do Brasil, Rio de Janeiro:Editora Helianthus, 2000.

UNITED STATES PATENT AND TRADEMARK OFFICE'S (USPTO). [Base de dados - Internet]. 2018. Disponível em: <http://patft.uspto.gov/netahtml/PTO/search-bool.html>. Acesso em: 14 maio 2018.

WORLD INTELLECTUAL PROPERTY ORGANIZATION (WIPO). [Base de dados - Internet]. 2018. Disponível em: <https://patentscope.wipo.int/search/pt/advancedSearch.jsf>. Acesso em: 14 maio 2018. 


\section{Sobre os autores}

\section{Cristiane Araujo Nascimento}

E-mail: cristianearaujonascimento@gmail.com

Doutoranda em Biotecnologia em Saúde pela Rede Nordeste de Biotecnologia - RENORBIO, Ponto Focal UFAL. Endereço profissional: Universidade Federal de Alagoas, Campus Arapiraca. Av. Manoel Severino Barbosa, s/n Bom Sucesso, Arapiraca, AL. CEP: 57309-005.

\section{Tatiane Luciano Balliano}

E-mail: tlb@qui.ufal.br

Doutora, pelo Instituto de Química e Biotecnologia da Universidade federal de Alagoas (IQB)/UFAL.

Endereço profissional: Campus A. C. Simões. Av. Lourival Melo Mota, s/n.

\section{Denise Macêdo Silva}

E-mail: denise.macedo15@hotmail.com

Graduanda em enfermagem, pela Universidade Federal de Alagoas (UFAL).

Endereço profissional: Avenida Manoel Severino Barbosa Rodovia, s/n - Bom Sucesso, Arapiraca, AL. CEP: 57309-005.

\section{Renan Macêdo Silva}

E-mail: renan.macedo50@hotmail.com

Graduando de Ciência da Computação.

Endereço profissional: Avenida Manoel Severino Barbosa Rodovia, s/n - Bom Sucesso, Arapiraca, AL. CEP: 57309-005.

\section{Karol Fireman Farias}

E-mail: karolfireman@hotmail.com

Doutora em Biotecnologia em Saúde, pela Universidade Federal de Alagoas (UFAL).

Endereço profissional: Avenida Manoel Severino Barbosa Rodovia, s/n - Bom Sucesso, Arapiraca, AL. CEP: 57309-005.

\section{Guilherme Benjamin Brandão Pitta}

E-mail: guilhermebbpitta@gmail.com

Doutor, pela Universidade Federal de Alagoas (Departamento de Medicina/UFAL).

Endereço profissional:Avenida Manoel Severino Barbosa Rodovia, s/n - Bom Sucesso, Arapiraca, AL. CEP: 57309-005. 